\title{
Mitochondrial Quality Control Governed by Ubiquitin
}

\author{
Sonia Ravanellit, Fabian den Brave ${ }^{2 t}$ and Thorsten Hoppe ${ }^{1,3 *}$ \\ ${ }^{1}$ Institute for Genetics and Cologne Excellence Cluster on Cellular Stress Responses in Aging-Associated Diseases, \\ University of Cologne, Cologne, Germany, ${ }^{2}$ Department of Molecular Cell Biology, Max Planck Institute of Biochemistry, \\ Martinsried, Germany, ${ }^{3}$ Center for Molecular Medicine Cologne, University of Cologne, Cologne, Germany
}

Mitochondria are essential organelles important for energy production, proliferation, and cell death. Biogenesis, homeostasis, and degradation of this organelle are tightly controlled to match cellular needs and counteract chronic stress conditions. Despite providing their own DNA, the vast majority of mitochondrial proteins are encoded in the nucleus, synthesized by cytosolic ribosomes, and subsequently imported into different mitochondrial compartments. The integrity of the mitochondrial proteome is permanently challenged by defects in folding, transport, and turnover of mitochondrial proteins. Therefore, damaged proteins are constantly sequestered from the outer mitochondrial membrane and targeted for proteasomal degradation in the cytosol via mitochondrial-associated degradation (MAD). Recent studies identified specialized quality control mechanisms important to decrease mislocalized proteins, which affect the mitochondrial import machinery. Interestingly, central factors of these ubiquitindependent pathways are shared with the ER-associated degradation (ERAD) machinery, indicating close collaboration between both tubular organelles. Here, we summarize recently described cellular stress response mechanisms, which are triggered by defects in mitochondrial protein import and quality control. Moreover, we discuss how ubiquitindependent degradation is integrated with cytosolic stress responses, particularly focused on the crosstalk between MAD and ERAD.

Keywords: C. elegans, mitochondria, proteostasis, mitochondria-associated degradation (MAD), ubiquitin, Cdc48, p97, Msp1

\section{INTRODUCTION}

Mitochondrial integrity relies on a sophisticated network of quality control machineries, which have been evolved to counteract challenges associated with the endosymbiotic integration of this organelle into eukaryotic cells (Youle, 2019). Along with a precise coordination between nuclear and mitochondrial gene expression (Couvillion et al., 2016), transcriptional stress response programs emerged as central mitochondrial surveillance mechanisms (Andréasson et al., 2019). Mitochondrial functionality is further supported by ubiquitin-dependent degradation of proteins accumulating under stress conditions. The outer and inner mitochondrial membrane (OMM and IMM) separate the lumen into the intermembrane space (IMS) and the matrix. Mitochondria are equipped with an elaborate set of proteases acting on the different sub-compartments, to maintain the mitochondrial proteome from the inside (Koppen and Langer, 2007; Quirós et al., 2015; Glynn, 2017). Otherwise, the integrity of the mitochondrial proteome is largely supported by the UPS 
localized in the cytosol (Franz et al., 2015; Bragoszewski et al., 2017; Braun and Westermann, 2017; D'Amico et al., 2017; Escobar-Henriques et al., 2020).

Besides the important role in ATP production and synthesis of amino acids, nucleotides, and iron-sulfur clusters (Lill and Mühlenhoff, 2008), mitochondria are also required for calcium buffering and apoptosis regulation (Wang and Youle, 2009; Contreras et al., 2010). Notably, mitochondria are tightly interconnected with other cellular organelles, especially the endoplasmic reticulum (ER) (Helle et al., 2013; van Vliet et al., 2014; Phillips and Voeltz, 2016). The contact between ER and mitochondria has been associated with aging and age-related diseases (Moltedo et al., 2019). In fact, the physical interaction between these tubular organelles supports the transfer of lipids, calcium ions and other metabolites, localizes to DNA nucleoids and regulates mitochondrial dynamics (Helle et al., 2013; Raturi and Simmen, 2013; van Vliet et al., 2014; Phillips and Voeltz, 2016; Moltedo et al., 2019). Recent studies suggest an intricate cooperation between ER and mitochondria in proteostasis (Dederer et al., 2019; Matsumoto et al., 2019), which constitute a newly developing research field promising for the development of therapeutic interventions as proposed for cardiac pathologies (Arrieta et al., 2020).

In this review we focus on quality control pathways that maintain mitochondrial functionality, involving cross-communication with the $\mathrm{ER}$ and other cellular compartments. We discuss recent discoveries on the role of the ubiquitin/proteasome-system (UPS) in mitochondrial quality control, including pathways that are constitutively active or triggered by metabolic stress to ensure mitochondrial integrity.

\section{PROTEIN DEGRADATION MECHANISMS}

Protein quality control is required at all steps originating from protein synthesis and involves a series of mechanisms dedicated to the surveillance of protein translation, transport, and turnover (Kaushik and Cuervo, 2015). Central players of the proteostasis network are molecular chaperones which mediate folding, targeting, and degradation of proteins (Klaips et al., 2018). The two major degradation pathways for proteins in the cytosol are the UPS and the autophagy-lysosomal pathway (Pohl and Dikic, 2019). In both proteolytic systems posttranslational attachment of the small polypeptide ubiquitin serves as a targeting signal for protein turnover. The modification with ubiquitin (ubiquitylation) is mediated by a three-step enzymatic cascade (Kerscher et al., 2006). First, the ubiquitinactivating enzyme (E1) forms a high-energy thioester bond between its catalytic cysteine and the $\mathrm{C}$-terminal glycine residue of ubiquitin, which is then transferred to a cysteine of an ubiquitin-conjugating enzyme (E2). The E2 cooperates with specific ubiquitin ligases (E3) to mediate the covalent attachment of ubiquitin mainly to a lysine residue in the selected substrate. Repeated cycles of this reaction either results in multiple monoubiquitylation of different lysine residues of a given substrate or formation of ubiquitin chains by targeting one of the seven lysines of ubiquitin (K6, K11, K27, K29, K33, K48, or K63) (Haakonsen and Rape, 2019). These different ubiquitindependent modifications termed "the ubiquitin code" serve as signals for different downstream events mediated by specialized binding proteins. Moreover, ubiquitylation can be reversed by different deubiquitylating enzymes (DUBs), which completely remove ubiquitin from substrates or trim ubiquitin chains to alter their composition (Clague et al., 2019). A prominent role of ubiquitylation is to target proteins for lysosomal or proteasomal turnover, which is often mediated by attachment of K48-linked ubiquitin chains (Dikic, 2017).

The turnover of soluble ubiquitylated proteins is mainly conducted by the $26 \mathrm{~S}$ proteasome (Bard et al., 2018). It constitutes a multicatalytic complex of a barrel-shaped core subunit known as $20 \mathrm{~S}$ proteasome and the $19 \mathrm{~S}$ regulatory particle, attached to one or both ends of the $20 \mathrm{~S}$ core. The regulatory particle is composed of a hexameric complex of AAA-ATPases that coordinates unfolding and translocation of the substrate into the core subunit. The $19 \mathrm{~S}$ subunit also contains scaffold proteins involved in substrate recognition and deubiquitylation as well as gate opening and binding with other external factors. The core subunit is composed of four stacked heptameric rings and contains the proteolytic activity required for the cleavage of unfolded polypeptides.

In contrast to soluble substrates, proteins organized in multimeric complexes or membrane bound, require an additional extraction step prior to proteasomal degradation. This function is mainly executed by Cdc48 (p97 or VCP in vertebrates), which belongs to the family of AAA-ATPases associated with diverse cellular activities (AAA+), commonly using ATP to perform mechanochemical reactions (Sauer and Baker, 2011; Glynn, 2017). In collaboration with substrate-specific cofactors, Cdc48 binds ubiquitylated proteins and targets them to the 26S proteasome (Franz et al., 2014; Barthelme and Sauer, 2016).

Larger structures such as aggregated proteins or organelles are targeted for degradation inside lysosomes (vacuole in yeast and plants), which contain promiscuous proteolytic enzymes for degradation of engulfed cargoes. In this process termed macroautophagy (hereafter autophagy), substrates are recognized by autophagy receptors bound to autophagosomal membranes, which triggers substrate engulfment by the autophagosomal membrane and subsequent lysosomal fusion (Khaminets et al., 2016). Similar to soluble misfolded proteins, protein aggregates are targeted for autophagic degradation by ubiquitylation, which is a common feature of the two proteolytic pathways ( $\mathrm{Lu}$ et al., 2017). Moreover, a specialized form of autophagy, called mitophagy, allows the selective turnover of entire mitochondria. This process can either be mediated by autophagy receptors residing in the OMM or by ubiquitylation of OMM proteins (Palikaras et al., 2018; Pickles et al., 2018).

\section{UBIQUITIN-DEPENDENT SURVEILLANCE OF THE MITOCHONDRIAL PROTEOME}

The degradation of ER-resident or mitochondrial proteins is regulated by two mechanistically similar ubiquitin-dependent 
pathways termed ER-associated degradation (ERAD) and mitochondria-associated degradation (MAD) (Heo and Rutter, 2011; Guerriero and Brodsky, 2012; Ruggiano et al., 2014; Braun and Westermann, 2017; Mehrtash and Hochstrasser, 2018). For both tubular organelles, proteins residing in the outer membrane are directly accessible for ubiquitylation. By contrast, proteins localized inside these organelles have to be transported across the membrane to be exposed to the ubiquitin-conjugation machinery. Cdc48 triggers ERAD by retro-translocation of substrate proteins out of the ER lumen and proteasomal degradation at the cytosolic side of the ER membrane. Therefore, substrates proteins are ubiquitylated by membrane-bound ubiquitin ligase complexes. These ERAD ligases interact with accessory factors such as the UBX-domain protein Ubx2 to recruit $\mathrm{Cdc} 48$ for coordinating substrate extraction and turnover (Neuber et al., 2005; Schuberth and Buchberger, 2005). Increasing evidence suggests that a comparable system employs the UPS in controlling quality control of mitochondrial proteins.

\section{UPS-Dependent Turnover of Mitochondrial Proteins}

The mitochondrial mass is efficiently regulated by the balanced coordination between biogenesis and degradation. In addition to the degradation of damaged mitochondrial proteins the UPS also contributes to remodeling of the mitochondrial proteome in response to metabolic changes (Figure 1) (Bragoszewski et al., 2017). Mitochondria form a highly dynamic network shaped by continuous fusion and fission events with adaptive morphology according to cellular needs. These fission and fusion events are mediated by large dynamin-like GTPases that provide the mechanical force to either fuse or separate membranes. In mammals, fission is induced by DRP1 (Dnm1 in yeast), whereas fusion is driven by mitofusins (Fzol in yeast) in the outer membrane and by OPA1 (Mgm1 in yeast) in the inner membrane (Escobar-Henriques and Anton, 2013). Fusion is known to enhance the exchange of important molecules and can temporarily compensate for defects in mitochondrial sub-populations. On the other hand, fission promotes mitochondrial motility and allows separation of damaged organelles for mitophagy (Lackner, 2014; Roy et al., 2015; Wai and Langer, 2016). Residing in the OMM, these GTPases present cytosolic domains which are targeted for ubiquitin-dependent degradation. Thus, the UPS provides a crucial role in the regulation of mitochondrial morphology and function (Bragoszewski et al., 2017).

Notably, the first identified MAD substrate was in fact the yeast mitofusin Fzol (Neutzner and Youle, 2005), which is embedded into the OMM by two transmembrane domains, exposing most of its amino acid residues to the cytosol. Ubiquitylation of Fzol mainly depends on the F-box protein Mdm30, which is part of the multi-subunit SCF (Skp, Cullin, Fbox) ubiquitin ligase complex (Fritz et al., 2003; EscobarHenriques et al., 2006; Cohen et al., 2008) (Table 1). The ubiquitin-modification of Fzo1 provides a regulatory role in the fusion of mitochondrial outer membranes, which is not necessarily linked to degradation (Anton et al., 2011; Cohen et al., 2011). In fact, proteasome-dependent turnover of Fzo1 largely depends on the nature of ubiquitylation, since the DUB Ubp2 is able to remove or trim down ubiquitin chains that are attached to Fzol, which regulates its degradation (Anton et al., 2013) (Table 1).

Depending on the physiological conditions, Cdc48 also appears to be required for Fzol regulation (Heo et al., 2010; Esaki and Ogura, 2012). Under oxidative stress conditions, Cdc48 together with its co-factors Vms1 and Npl4 facilitates Fzo1 degradation (Heo et al., 2010). Under non-stressed conditions Fzo1 and additional other OMM proteins are targeted by another Cdc48 complex involving Npl4, Ufd1, and Doa1, also called Ufd3 (Wu et al., 2016) (Figure 1 and Table 1). This might however reflect protein quality control triggered by experimental tagging of the membrane-bound proteins, since non-tagged endogenous Fzol is rather stabilized by Cdc48, which exerts a regulatory role during OMM fusion (Simões et al., 2018; Anton et al., 2019). Turnover of mitofusins by MAD is widely conserved among species including Marf in flies (Ziviani et al., 2010; Wang et al., 2016) and Mfn1/2 in mammals (Tanaka et al., 2010; Chan et al., 2011; Xu et al., 2011). A common role of MAD in this case is the inhibition of mitochondrial fusion by mitofusin degradation, resulting in mitochondrial fragmentation. In mammals, the E3 ligases Parkin and MARCH5 mediate p97 dependent extraction and proteasomal turnover of mitofusins, inducing mitochondrial fission and mitophagy (Karbowski et al., 2007; Ziviani et al., 2010; Chan et al., 2011; Xu et al., 2011; Wang et al., 2016). Moreover, MAD was observed to play a role in regulation of apoptosis, since it targets the anti-apoptotic BCL2 protein MCL1 for degradation in mammals (Inuzuka et al., 2011; Xu et al., 2011).

Besides Cdc48, Msp1 (ATAD1 in humans) supports extraction and degradation of mitochondrial proteins (Figure 1 and Table 1). Despite being an AAA-ATPase similar to Cdc48, Msp1 contains a membrane spanning domain at the $\mathrm{N}$-terminus and is mainly localized at the OMM, but is also attached to peroxisomes (Nakai et al., 1993; Chen et al., 2014; Okreglak and Walter, 2014). Msp1/ATAD1 plays a key role in mitochondrial proteostasis since it mediates the degradation of mislocalized tail-anchored proteins (TA-proteins) from the OMM. A baseline degradation of TAproteins by the UPS occurs to maintain a dynamic stationary level and ensure insertion into the outer membrane of the correct organelle (Chen et al., 2014; Okreglak and Walter, 2014). Moreover, TA-proteins destined to the ER can mislocalize to the OMM, when their import system, termed the GET pathway in yeast and TRC in mammals, is impaired. These mislocalized TA-proteins are recognized by Msp1 at the OMM and targeted for proteasomal degradation. For instance, the TA-protein Pex15 is partly inserted in the OMM, even in the presence of the fully functional GET pathway, suggesting that TA-proteins are constitutively inserted into the OMM, where they have to be removed by Msp1 (Chen et al., 2014; Okreglak and Walter, 2014). Recently, a similar role of Msp1 was described at peroxisomes (Weir et al., 2017). Of note, the proposed ATP-dependent activity of Msp1 in the extraction of TA-proteins from mitochondrial membranes was confirmed in an in vitro system of reconstituted proteoliposomes (Wohlever et al., 2017).

Surprisingly, mistargeted TA-proteins extracted by Msp1 are ubiquitylated by Doa10, an E3 ligase residing in the ER membrane and degraded in a Cdc48-dependent manner 


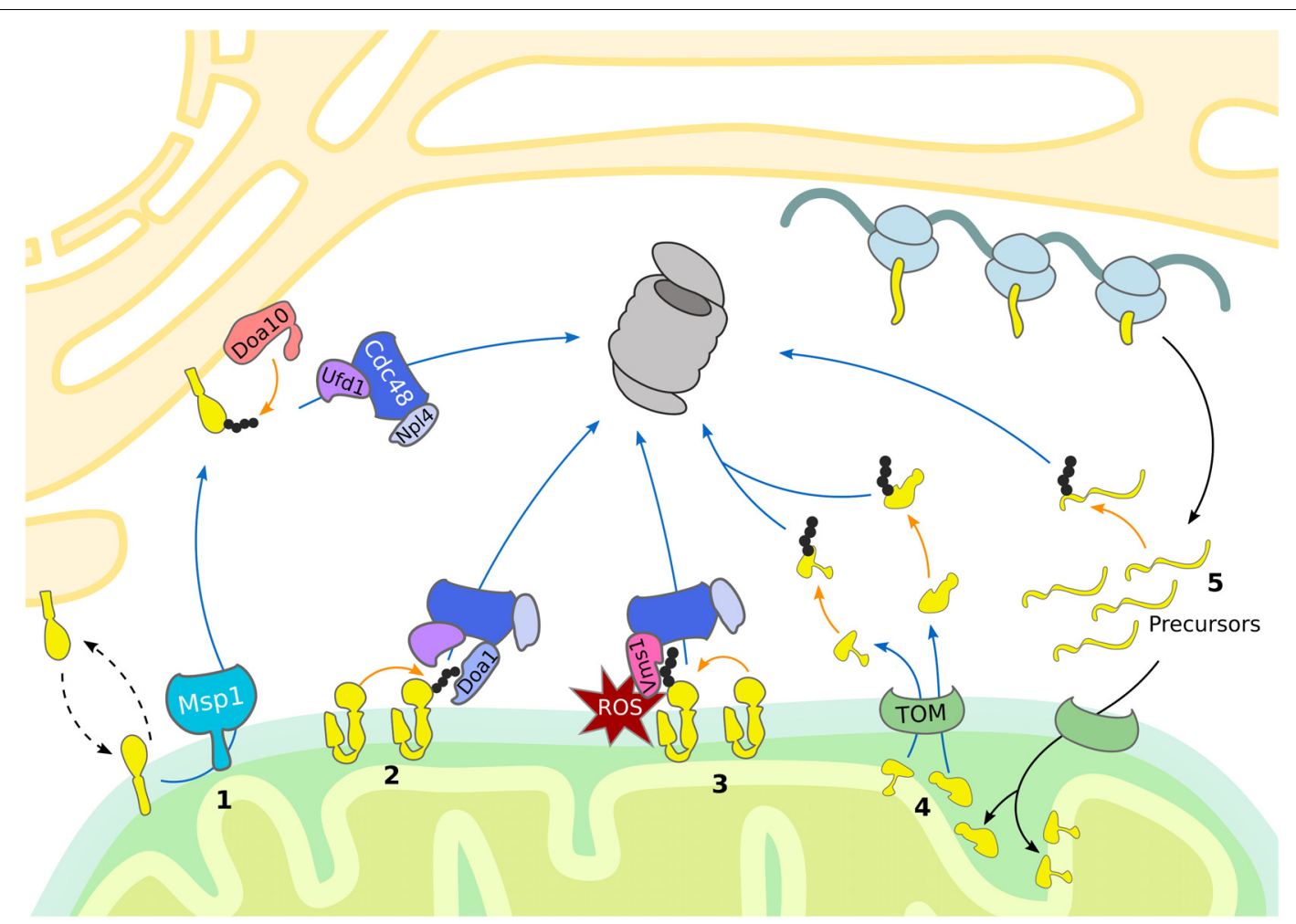

FIGURE 1 | UPS-dependent turnover of mitochondrial proteins. The cytosolic UPS mediates mitochondrial protein turnover by ubiquitylation (orange arrows) and targeting (blue arrows) of substrates for degradation by the 26S proteasome. (1) Mislocalized tail-anchored proteins are extracted from the OMM by Msp1, ubiquitylated by the ER-associated E3 ubiquitin ligase Doa10 and then translocated to the proteasome by the Cdc48Ufd1/Npl4 complex. (2, 3) Degradation of OMM proteins occurs via Cdc48-dependent translocation to the $26 \mathrm{~S}$ proteasome. (2) Upon oxidative stress, Vms 1 translocates to the OMM where it recruits Cdc48 and its co-factor Npl4. (3) Under normal conditions OMM proteins are ubiquitylated and translocated to the $26 \mathrm{~S}$ proteasome by Cdc48 together with the co-factors Ufd1, Npl4, and Doa1/Ufd3. (4) Proteins residing in the IMS and IMM are retro-translocated via the TOM complex into the cytosol for ubiquitin-dependent proteasomal degradation. (5) Prior import, mistargeted or damaged mitochondrial precursor proteins are degraded by the UPS.

(Figure 1 and Table 1). This initial observation suggests a role of ERAD in the turnover of OMM proteins (Dederer et al., 2019; Matsumoto et al., 2019). Indeed, Msp1 co-localizes with its substrates at ER-mitochondria contact sites, which however, seem to be dispensable for Doa10/Cdc48 dependent turnover of Msp1 substrates (Matsumoto et al., 2019). Therefore, it was proposed that Msp1 extracted TA-proteins are inserted into the ER membrane for Doa10-mediated ubiquitylation. Subsequently, Cdc48 is recruited together with the cofactors Ufd1 and Npl4 to translocate mistargeted TA-proteins to the 26S proteasome (Matsumoto et al., 2019). Intriguingly, Msp1 appears to extract only monomeric proteins and not multi-complexes, suggesting that the recognition of mistargeted TA-protein is based on the weak interaction with the membrane of a single transmembrane domain (Dederer et al., 2019). Interestingly, Msp1 emerged as an MAD substrate itself, whose degradation depends on the Doa1Cdc48-Ufd1-Npl4 complex (Wu et al., 2016). This might indicate a role of the UPS in the regulation of mitochondrial protein half-life by controlling Msp1 level and extraction of TA-proteins.

Intriguingly, besides membrane bound proteins also inner mitochondrial proteins have been reported to be ubiquitylated. In analogy to ERAD, retro-translocation of mitochondrial proteins across IMM and/or OMM has been suggested to enable ubiquitylation at the cytosolic surface of the organelle (Figure 1). Indeed, such activity has been demonstrated for IMS proteins, which upon unfolding, translocate into the cytosol (Bragoszewski et al., 2015). The export of these proteins seems to depend on the translocase of the outer membrane (TOM) complex also required for import, however, further studies are required to investigate the exact mechanism of mitochondrial retrotranslocation. Notably, this degradation of IMS proteins appears to be utilized to rewire the mitochondrial proteome to changing metabolic conditions upon shift from respiration to fermentation (Bragoszewski et al., 2015). A major challenge in exploring this novel mechanism is to carefully distinguish ubiquitin-dependent degradation of retro-translocated mitochondrial protein versus cytosolic precursor proteins that accumulate when mitochondrial import is impaired (Figure 1).

\section{Mitochondrial Import Control}

Nuclear-encoded mitochondrial proteins are synthetized as precursors in the cytosol and subsequently imported and sorted to different mitochondrial compartments. After passing through the TOM complex, precursor proteins are redistributed to their final intra-mitochondrial destination. At least five distinct but interconnected import pathways have been identified, each one 
TABLE 1 | Regulators of ubiquitin-dependent mitochondrial quality control.

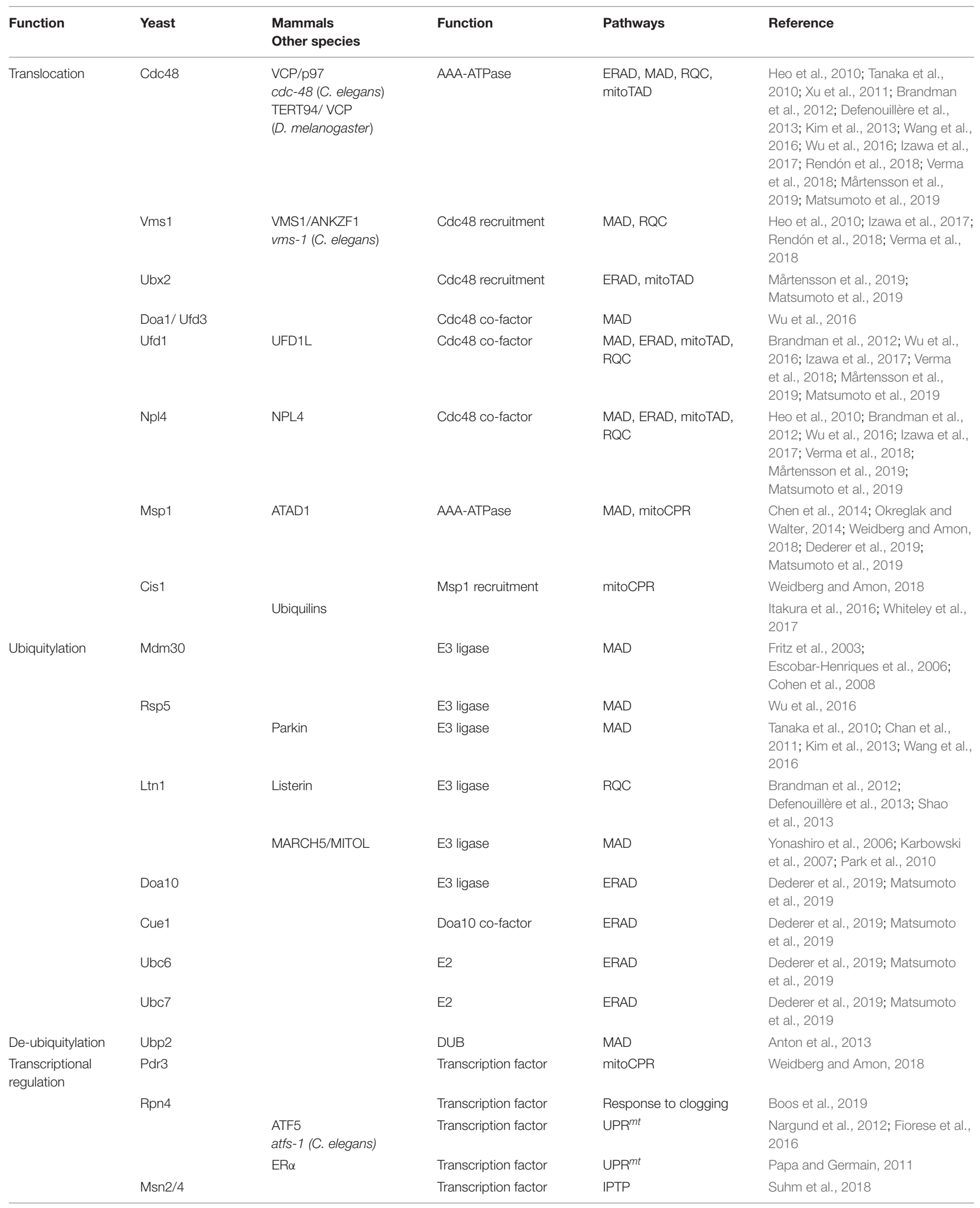




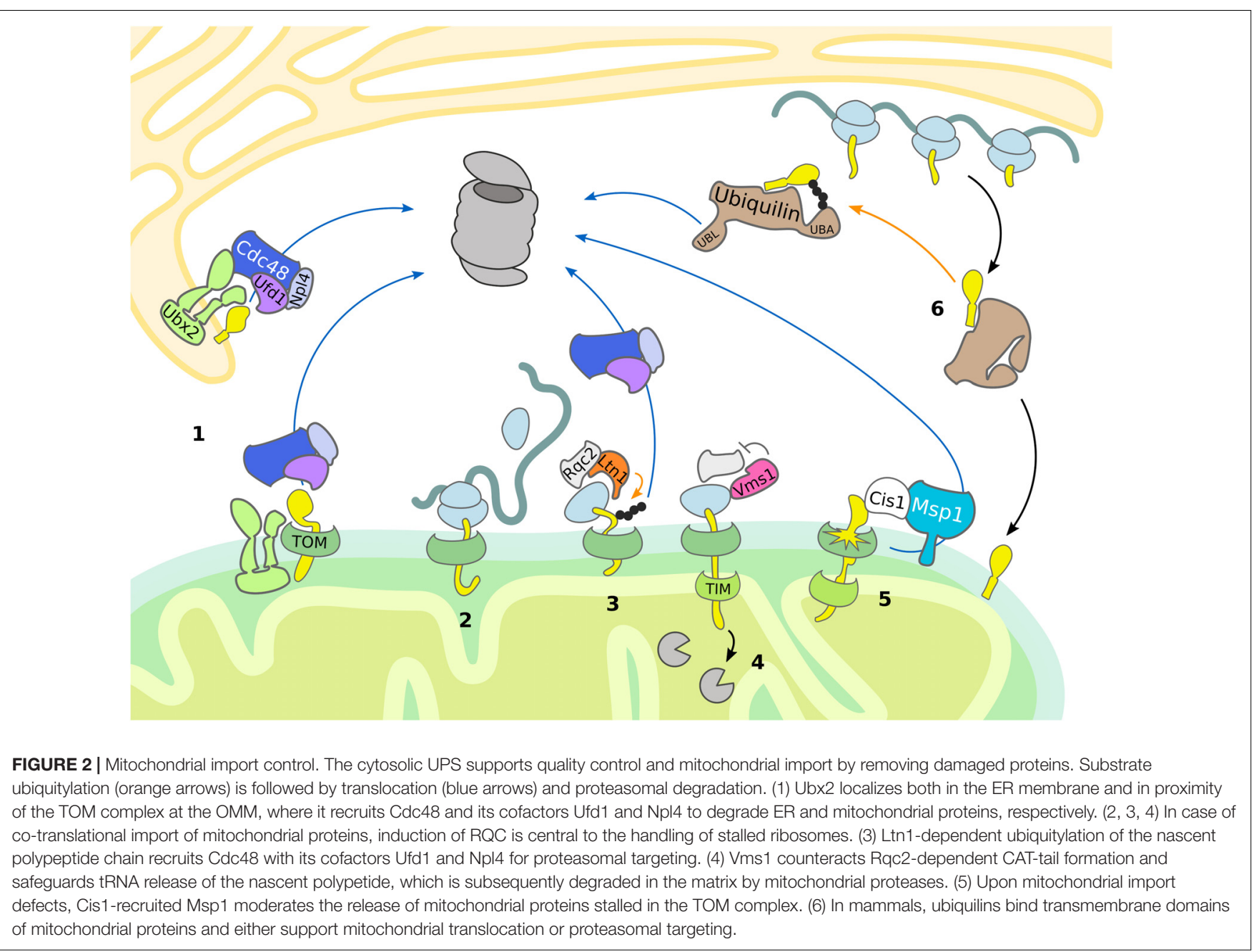

directed by a specific targeting signal (Neupert and Herrmann, 2007; Chacinska et al., 2009; Wiedemann and Pfanner, 2017; Pfanner et al., 2019). In order to avoid the import of aberrant proteins into mitochondria and to ensure mitochondrial proteostasis, specialized surveillance mechanisms monitor the nuclear-encoded proteins before and during mitochondrial import (Figures 1, 2).

Interestingly, it was shown that mitochondrial precursor proteins are constantly degraded in a ubiquitin-dependent manner. For example, both the wild-type and mutant IMS protein endonuclease $G$ (endoG) are degraded by the UPS before their import (Radke et al., 2008). However, in contrast to the mutant form, wild-type endoG can be alternatively degraded by the IMS protease Omi, supporting the idea that the proteasome plays a role in degradation of defective mitochondrial proteins prior to import. In line with this conclusion, the UPS was reported to assist the import of intermembrane proteins by constantly degrading precursor proteins before they enter mitochondria (Bragoszewski et al., 2013; Kowalski et al., 2018) (Figure 1).

In mammalian cells, quality control of membrane proteins recruited to mitochondria is provided by ubiquilins
(Itakura et al., 2016; Whiteley et al., 2017), which are substrate receptors supporting proteasomal turnover. They typically possess a ubiquitin-binding UBA domain for recognition of ubiquitylated substrates and a ubiquitin-like UBL domain for proteasome targeting. Ubiquilins bind to mitochondrial proteins containing transmembrane domains, thereby preventing their aggregation (Itakura et al., 2016). As long as the substrate remains unmodified, the UBA domain of ubiquilin is bound to its own UBL domain, promoting mitochondrial import. Upon substrate ubiquitylation, the UBL domain is released, which results in targeting the mitochondrial substrate proteins for proteasomal degradation. Thus, ubiquilins exert an important triage function regulating mitochondrial import (Figure 2).

Mitochondrial targeting and protein import are governed at the mitochondrial import channel by a newly proposed MAD pathway. A subpopulation of Ubx2 binds to the TOM complex, which recruits Cdc48 to initiate degradation of partially imported proteins similarly to ERAD (Mårtensson et al., 2019) (Figure 2 and Table 1). This mechanism is termed mitochondrial protein translocation-associated degradation (mitoTAD). Interestingly, Ubx2 binding with Cdc48 depends on the co-factor Ufd1, whereas the other Cdc48 partners implicated in MAD, Vms1 and 
Doa1/Ufd3, are not involved in mitoTAD. However, combined deletion of Ubx2 with either Vms1 or Msp1 caused strong mitochondrial defects and an increase of ubiquitylated proteins bound to the TOM complex, suggesting redundant roles of the described mitochondrial import control pathways (Mårtensson et al., 2019). Indeed, Msp1 has been reported to function in degrading substrates from the TOM channel as well (Weidberg and Amon, 2018). Mitochondrial import stress induces the expression of Cis1, which mediates the recruitment of Msp1 to the import pore (Weidberg and Amon, 2018; Boos et al., 2019). Subsequently, Msp1 triggers proteasomal turnover of proteins clogging the TOM complex, thereby maintaining mitochondrial protein import and proteostasis (Figure 2) (Weidberg and Amon, 2018).

\section{Ribosome-Associated Quality Control of Mitochondrial Proteins}

The first contact of mitochondrial proteins with the cytosolic proteostasis network occurs already during translation at cytosolic ribosomes. It was recently shown that stalling of ribosomes, which indicates aberrant mRNA, defective ribosome assembly, or an accumulation of nascent protein, triggers ribosome-associated quality control (RQC) (Brandman and Hegde, 2016; Joazeiro, 2019). Stalled ribosomes are sensed by the yeast RQC complex subunit Rqc2 (NEMF in mammals), recruiting the E3 ligase Ltn1 (listerin in mammals) for polyubiquitylation of the nascent peptide. The polyubiquitin chain serves as signal to attract Cdc48 and its cofactors Ufd1 and Npl4 (UFD1 and NPLOC4 in mammals), which together shuttle the polypeptide to the proteasome. In case the accessible nascent polypeptide does not contain any lysine residues, Rqc2 can induce a peptide extension by addition of a C-terminal alanine and threonine (CAT) tail. Through this elongation, more residues of the nascent chain get exposed outside of the ribosome exit tunnel, until a lysine becomes available for Ltn1 dependent ubiquitylation. In addition, CAT-tails have been reported to induce aggregation of proteins, which might act as a protective mechanism in case of RQC failure.

Since the targeting sequence of many mitochondrial proteins is at the N-terminus, import can occur either posttranslationally or co-translationally (Neupert and Herrmann, 2007; Wiedemann and Pfanner, 2017). If import and translation occur simultaneously, part of the nascent polypeptide chain is sequestered inside the mitochondrial import machinery before completion of translation and release from the ribosome. Thus, in contrast to cytosolic proteins, ubiquitylation of mitochondrial proteins emerging at the ribosome might fail due to the close proximity between the ribosome and the mitochondrial translocation machinery and reduced accessibility of the co-translationally imported nascent polypeptide chain. Consequently, Rqc2 dependent CAT tailing can induce protein aggregation inside the mitochondrial matrix and subsequent degeneration of the mitochondrial respiratory capacity (Izawa et al., 2017). The formation of protein aggregates is counteracted by Vms1 (ANKZF1 in mammals) activity, which mediates the release of nascent polypeptides from the tRNA and thereby terminates CAT tail formation (Izawa et al., 2017; Rendón et al., 2018; Verma et al., 2018) (Figure 2 and Table 1).

\section{MITOCHONDRIAL STRESS RESPONSE MECHANISMS}

Based on the intricate network of ubiquitin-dependent quality control mechanisms, stressed or defective mitochondria often affect UPS activity, which largely relies on the abundance and activity of the 26S proteasome (Marshall and Vierstra, 2019). Central to this regulatory relationship are reactive oxygen species (ROS), that are generated inside mitochondria via oxidative phosphorylation (Lefaki et al., 2017). Changes in ROS level have been linked to reversible disassembly of the $26 \mathrm{~S}$ proteasome into $20 \mathrm{~S}$ and $19 \mathrm{~S}$ subunits. These changes in proteasomal composition and activity might reflect a protective strategy by favoring ATP/ubiquitin-independent degradation of oxidized proteins via 20S proteasomes (Wang et al., 2010; Grune et al., 2011; Livnat-Levanon et al., 2014). In C. elegans, mitochondrial impairment was linked to defective turnover of cytosolic UPS model substrates despite no increase in ROS, suggesting the existence of a distinct response mechanisms regulating UPS activity (Segref et al., 2014). Mitochondrial translation accuracy has been as well-linked to cytosolic proteostasis (Suhm et al., 2018). Particularly, mitochondrial ribosome mutations in yeast either improving or reducing translation accuracy showed an increased or decreased turnover of a cytosolic proteasome substrate, respectively. However, in both studies, proteasome activity was not altered in comparison to wild-type controls, suggesting that the regulation occurs upstream of proteasomal degradation (Segref et al., 2014; Suhm et al., 2018).

Interestingly, recent studies have demonstrated that specialized stress response pathways induced by mitochondrial impairment affect the UPS by regulating gene expression in the nucleus and protein translation in the cytosol (Papa and Germain, 2011; Nargund et al., 2012; Wrobel et al., 2015; Weidberg and Amon, 2018; Boos et al., 2019). Along with the well-known heat shock response (HSR) in the cytosol (Richter et al., 2010) and the unfolded protein response (UPR) in the ER (Walter and Ron, 2011), a mitochondrial UPR (UPR ${ }^{\mathrm{mt}}$ ) has been thoroughly investigated (Münch, 2018; Shpilka and Haynes, 2018). These three stress response mechanisms are characterized by one or more signal transduction pathways that activate transcription of protective genes, encoding molecular chaperones, proteases, and UPS components. Consequently, induction of HSR, UPR, and $\mathrm{UPR}^{\mathrm{mt}}$ supports proteostasis by inducing UPS function.

Even though first detected in mammalian cells (Martinus et al., 1996; Zhao et al., 2002), the UPR ${ }^{\mathrm{mt}}$ mechanism has been characterized in C. elegans (Yoneda, 2004; Haynes et al., 2010; Nargund et al., 2012). Central to this mitochondrial stress response is the transcription factor ATFS-1, which translocates into the nucleus when mitochondrial import is blocked (Nargund et al., 2012). Although the downstream transcriptional regulation induced in case of mitochondrial stress is similar to C. elegans, the regulatory signaling of the mammalian $U P R^{m t}$ emerged 


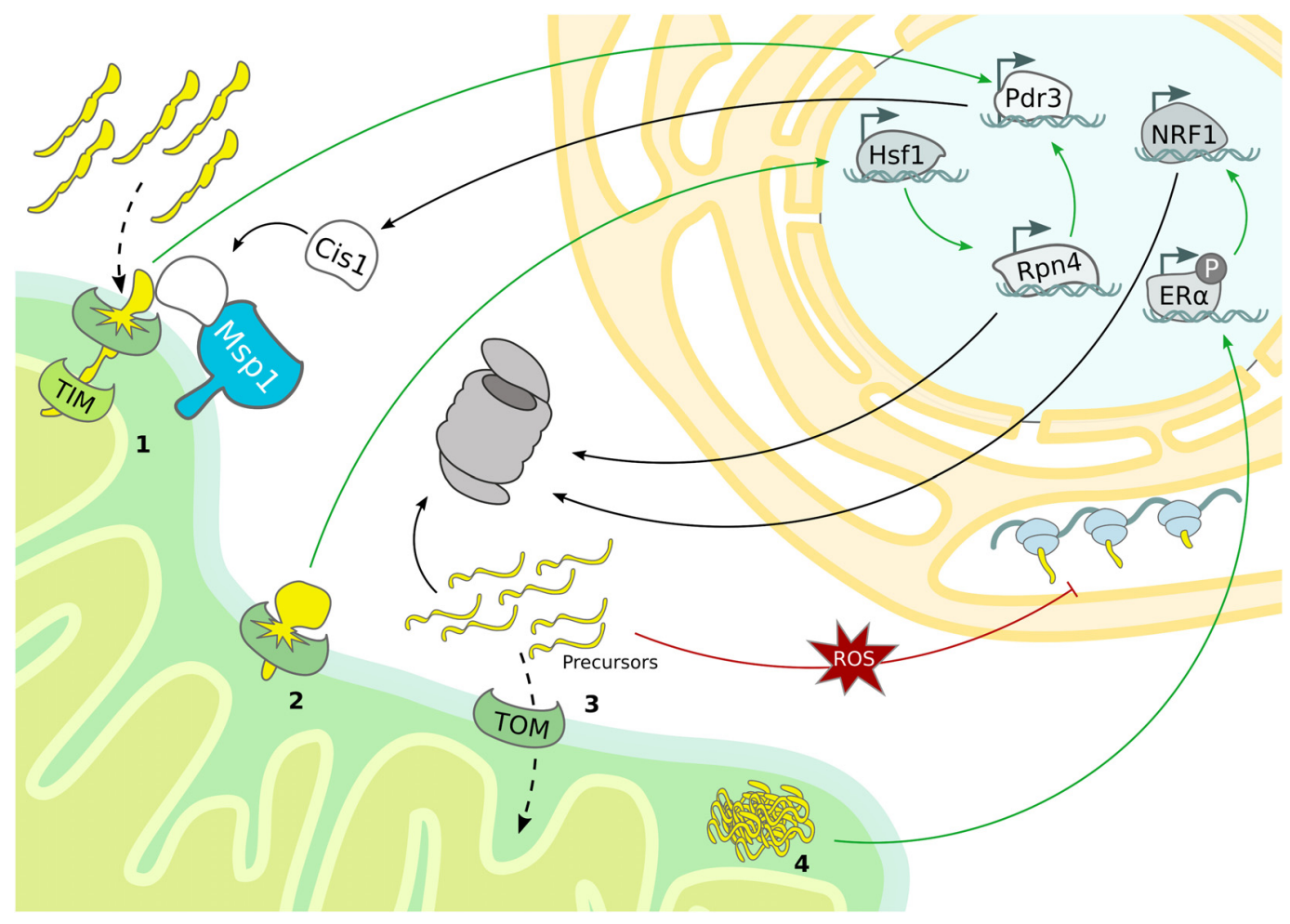

FIGURE 3 | Mitochondrial stress response mechanisms. Mitochondrial stress pathways regulate nuclear gene transcription and cytosolic protein translation to sustain proteostasis. (1) Overexpression of bipartite signal-containing proteins activates the mitoCPR, which triggers Pdr3-dependent expression of MDR genes, from which Cis1 promotes the recruitment of Msp1 to the TOM complex. (2) TOM clogging activates Hsf1-dependent expression of Rpn4, which drives expression of proteasomal subunits and mitoCPR-induced Pdr3. (3) The accumulation of mitochondrial precursor proteins caused by import defects boosts proteasomal activity and depletes general protein translation in the cytosol. (4) In mammals, protein aggregation in the IMS induces proteasome activity by ligand-independent activation of $\mathrm{ER} \alpha$ and subsequent upregulation of NRF1.

to be more complex and a general consensus model is still missing (Münch, 2018). Notably, a specialized mammalian $\mathrm{UPR}^{\mathrm{mt}}$ was reported to enhance proteasomal activity in response to protein aggregation in the IMS (Papa and Germain, 2011). The proposed molecular mechanism is based on the ligandindependent activation of the estrogen receptor alpha (ER $\alpha)$, which upregulates NRF1, a transcription factor involved in the expression of proteasomal subunits (Figure 3 and Table 1) (Papa and Germain, 2011). Accordingly, the C. elegans NRF homolog SKN-1 has also been identified as a downstream target of the $\mathrm{UPR}^{\mathrm{mt}}$ (Nargund et al., 2012). In yeast, increased proteasome activity was detected in consequence of mitochondrial import defects, indicating an UPR activated by the mistargeting of proteins (UPR ${ }^{\mathrm{am}}$ ) (Wrobel et al., 2015). Thus, defective mitochondrial import generates an accumulation of misfolded proteins in the cytosol, which aggravates proteasomal assembly and proteolytic activity (Figure 3).

The use of deep transcriptome sequencing, combined with proteomics allowed to monitor transcriptional and translational changes over time after 'clogging' of the TOM complex in yeast. Under these conditions the heat shock responsive transcription factor Hsf1 is activated and triggers the expression of molecular chaperones. Interestingly, one Hsf1-dependent target gene encodes the transcription factor Rpn4, which specifically upregulates proteasomal subunits, constituting a second layer of response that is activated upon prolonged stress (Boos et al., 2019). In addition to Hsf1 activation, the expression of nuclear encoded respiratory chain subunits is downregulated, which might serve to reduce the mitochondrial import load (Figure 3). Another transcriptional stress response activated by import defects is the mitochondrial compromised protein import response (mitoCPR) (Weidberg and Amon, 2018). Here, import inhibition caused by overexpression of bipartite signal-containing proteins, which are normally inserted into the IMS, provokes expression of multi drug resistance (MDR) response genes by the transcription factor Pdr3. One of the most upregulated genes is Cis1, which is recruited to the OMM and, by interacting with Tom70 and Msp1, supports the Msp1 and proteasome-dependent degradation of non-imported mitochondrial precursor proteins. Interestingly, the correct functionality of this pathway is fundamental for cell survival upon defective mitochondrial import but not under normal growth conditions (Figure 3 and Table 1) (Weidberg and Amon, 2018).

Although a general transcriptional program has not been identified yet, an intricate cooperation of the different quality control pathways becomes evident. For example, Rpn4 is not only initiated upon TOM "clogging," but also in import-defective 


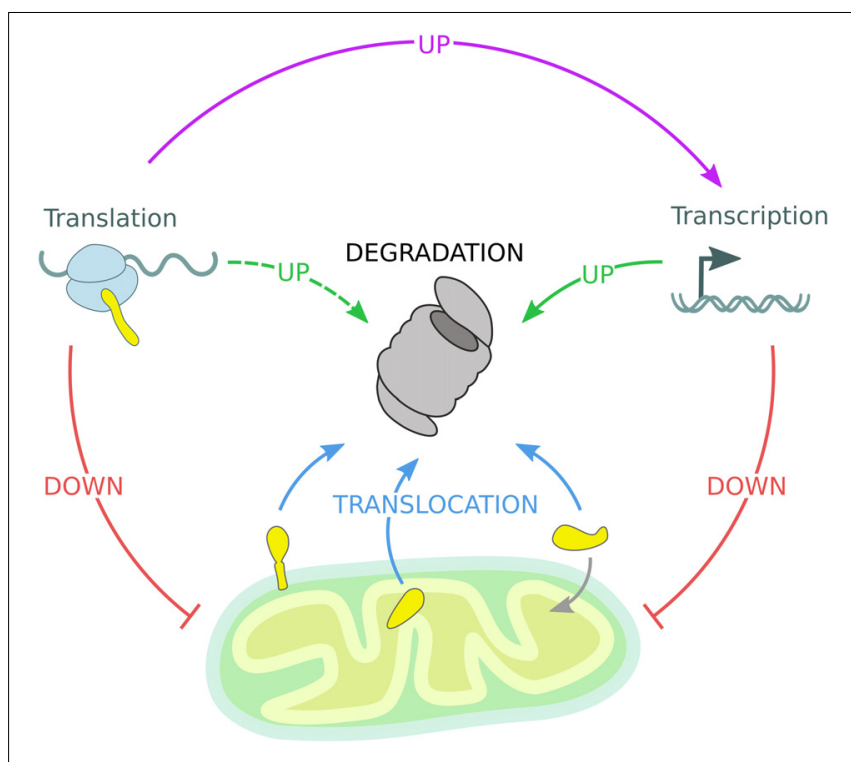

FIGURE 4 | Mitochondrial quality control governed by the UPS. The mitochondrial proteome is regulated by constant degradation of mitochondrial proteins, which are sequestered from the organelle and translocated (blue arrows) to the $26 \mathrm{~S}$ proteasome for degradation. In addition, mitochondrial precursor proteins are degraded by the $26 \mathrm{~S}$ proteasome if not efficiently imported into mitochondria. Upon mitochondrial stress, transcription and translation of mitochondrial genes are diminished, reducing the substrate load of both the mitochondrial import machinery and the 26S proteasome (red arrows). Moreover, transcription of protective genes is induced to enhance UPS activity (solid green arrow). Stress-induced inhibition of global protein translation triggers the expression of specialized transcription factors responsible for the activation of inducible stress response programs mentioned before (violet arrow), while other gene products might support UPS function (dashed green arrow).

yeast mutants related to $\mathrm{UPR}^{\mathrm{am}}$ (Wrobel et al., 2015; Boos et al., 2019). However, in contrast to clogging the TOM channel (Boos et al., 2019), import-defects do not induce the expression of proteasome subunits but of proteasome assembly chaperones (Wrobel et al., 2015). These discrepancies suggest that Rpn4 might be activated in case of mitochondrial defects, but probably distinct transcriptional programs characterize specific types of import-related stress. Moreover, the Pdr3dependent transcriptional induction of Cis1 upon accumulation of bipartite signal-containing proteins also requires Rpn4 (Figure 3) (Boos et al., 2019).

An additional cellular strategy to avoid UPS overload under prolonged mitochondrial stress conditions is based on suppression of protein translation both inside and outside of the organelle (Münch, 2018; Samluk et al., 2018; Shpilka and Haynes, 2018). The predominant mechanism of cytosolic translation inhibition is mediated by phosphorylation of the eukaryotic transition initiation factor 2 alpha (eiF $2 \alpha$ ), which prevents formation of the translation initiation complex (Sonenberg and Hinnebusch, 2009). However, oxidative stress generated upon dysfunctional mitochondrial import was recently reported to reduce cytosolic translation independently of eiF $2 \alpha$ phosphorylation. For instance, the translation machinery can be directly modulated by the redox status of proteins participating in translation (Figure 3) (Topf et al., 2018). Besides reducing global protein translation, mitochondrial import defects have also been proposed to induce translation of particular mRNAs (Wang and Chen, 2015; Topf et al., 2016). Specialized translation of stress-related transcription factors has already been described as a downstream event of eiF $2 \alpha$ phosphorylation, which therefore trigger numerous stress responses, including the UPR ${ }^{\mathrm{mt}}$ (Fiorese et al., 2016; Pakos-Zebrucka et al., 2016; Quirós et al., 2017).

Even though a common mechanism has not been identified yet, it is obvious that diverse, overlapping mitochondrial signaling pathways are activated to support proteostasis and cellular survival. These pathways influence gene expression to adapt mitochondrial and cytosolic protein degradation pathways under mitochondrial stress conditions.

\section{CONCLUSION}

Each cellular sub-compartment is equipped with specialized quality control machineries, which are intricately connected and cross-communicate. The reported studies support the idea that ubiquitin-dependent mitochondrial quality control pathways efficiently adapt in response to environmental and metabolic changes. However, in case of acute stress conditions, specialized response programs are induced to adjust the UPS capacity and thereby restore organellar proteostasis (Pakos-Zebrucka et al., 2016; Braun and Westermann, 2017; D’Amico et al., 2017; Pickles et al., 2018; Andréasson et al., 2019; Zheng et al., 2019; Escobar-Henriques et al., 2020). Besides understanding the mechanistic details of individual pathways, the regulation of cell-type specific and organismal composition of mitochondrial quality control need to be further addressed. Mechanistically, ubiquitindependent mitochondrial proteostasis follows a series of common regulatory events: stress sensing, substrate targeting and modification, protein translocation, and proteasomal degradation. Mitochondrial quality control is further regulated by nuclear gene transcription and cytosolic protein translation events (Figure 4). Overall, these proteostasis strategies are highly adaptive and can efficiently and dynamically modulate the stability of the mitochondrial proteome according to cellular needs.

\section{FUTURE PERSPECTIVES}

Regulation of mitochondrial quality control by the UPS emerged to be conserved in all eukaryotes, involving Cdc48/p97 and the $26 \mathrm{~S}$ proteasome. However, mechanistic details on substrate selection and ubiquitin ligases remain largely unclear. In contrast to proteasomal degradation of OMM proteins, little is known about the turnover of intra-mitochondrial proteins. Especially how mitochondrial substrates are retro-translocated from the different inner mitochondrial compartments into the cytosol is of central importance for understanding the regulation of MAD. Conversely, a novel functional role of the mitochondrial translocation machinery has been identified, which seems to 
import cytosolic aggregation-prone proteins into mitochondria for efficient degradation (Ruan et al., 2017). A more detailed view on the protein shuttling between mitochondria and cytosol will extend our current view on the cellular mechanisms dedicated to proteostasis maintenance and the reciprocal role of mitochondrial and cytosolic proteolytic systems.

Besides the high degree of mechanistic similarities between the MAD and ERAD pathways, mitochondrial quality control is further defined by functional mitochondria-ER interactions. For example, substrates extracted from the OMM by Msp1 are targeted to the ER for subsequent proteasomal degradation (Dederer et al., 2019; Matsumoto et al., 2019). Moreover, mitochondrial precursor proteins have been identified to associate with the ER membrane before being imported. The recently proposed ER-surface mediated targeting (ER-SURF) model describes the association of mitochondrial precursor proteins with the ER membrane and their rerouting to mitochondria by the ER-localized chaperone Djp1 (Hansen et al., 2018). Thus, the interaction between mitochondria and ER might play a conceptual role in protein quality control of mitochondrial proteins.

Mitochondrial impairments have been associated to several pathologies not only limited to metabolic diseases or myopathies, but including cancer (Vyas et al., 2016; Denisenko et al., 2019), pulmonary hypertension (Chen et al., 2019) and neurodegenerative disorders such as Alzheimer's and Parkinson's

\section{REFERENCES}

Andréasson, C., Ott, M., and Büttner, S. (2019). Mitochondria orchestrate proteostatic and metabolic stress responses. EMBO Rep. 20:e47865. doi: 10. 15252/embr.201947865

Anton, F., Dittmar, G., Langer, T., and Escobar-Henriques, M. (2013). Two deubiquitylases act on mitofusin and regulate mitochondrial fusion along independent pathways. Mol. Cell 49, 487-498. doi: 10.1016/j.molcel.2012. 12.003

Anton, F., Fres, J. M., Schauss, A., Pinson, B., Praefcke, G. J. K., Langer, T., et al. (2011). Ugo1 and Mdm30 act sequentially during Fzo1-mediated mitochondrial outer membrane fusion. J. Cell. Sci. 124, 1126-1135. doi: 10.1242/jcs. 073080

Anton, V., Buntenbroich, I., Schuster, R., Babatz, F., Simões, T., Altin, S., et al. (2019). Plasticity in salt bridge allows fusion-competent ubiquitylation of mitofusins and Cdc48 recognition. Life Sci. Alliance 2:e201900491. doi: 10. 26508/lsa.201900491

Arrieta, A., Blackwood, E. A., Stauffer, W. T., and Glembotski, C. C. (2020). Integrating ER and mitochondrial proteostasis in the healthy and diseased heart. Front. Cardiovasc. Med. 6:193. doi: 10.3389/fcvm.2019.00193

Bard, J. A. M., Goodall, E. A., Greene, E. R., Jonsson, E., Dong, K. C., and Martin, A. (2018). Structure and function of the 26 S proteasome. Annu. Rev. Biochem. $87,697-724$.

Barthelme, D., and Sauer, R. T. (2016). Origin and functional evolution of the Cdc48/p97/VCP AAA + protein unfolding and remodeling machine. J. Mol. Biol. 428, 1861-1869. doi: 10.1016/j.jmb.2015.11.015

Boos, F., Krämer, L., Groh, C., Jung, F., Haberkant, P., Stein, F., et al. (2019). Mitochondrial protein-induced stress triggers a global adaptive transcriptional programme. Nat. Cell Biol. 2019, 442-451. doi: 10.1038/s41556-019-0294-5

Bragoszewski, P., Gornicka, A., Sztolsztener, M. E., and Chacinska, A. (2013). The ubiquitin-proteasome system regulates mitochondrial intermembrane space proteins. Mol. Cell. Biol. 33:2136. doi: 10.1128/MCB.01579-12

Bragoszewski, P., Turek, M., and Chacinska, A. (2017). Control of mitochondrial biogenesis and function by the ubiquitin - proteasome system. Open Biol. 7:170007. doi: 10.1098/rsob.170007 disease (Kim and Mook-Jung, 2019; Tapias, 2019). Thus, further understanding of mitochondrial surveillance mechanisms might help to establish therapeutic interventions for treatment of mitochondrial pathologies. The multiple layers of mitochondrial regulation that can lead to disease progression if defective makes mitochondrial quality control a challenging but exciting research field, which is more and more integrated in the context of specialized cellular pathways, from basic research to clinical studies.

\section{AUTHOR CONTRIBUTIONS}

All authors conceived, wrote the manuscript, and critically revised the manuscript. SR designed the figures.

\section{ACKNOWLEDGMENTS}

We thank the members of our laboratory for critical discussion and helpful advice on the manuscript. We apologize for not having cited valuable contributions due to size limitation. This work is supported by the Deutsche Forschungsgemeinschaft (DFG, German Research Foundation) - SFB 1218 Projektnumber 269925409 and Cluster of Excellence EXC 229/ CECAD to TH.

Bragoszewski, P., Wasilewski, M., Sakowska, P., Gornicka, A., Böttinger, L., Qiu, J., et al. (2015). Retro-translocation of mitochondrial intermembrane space proteins. PNAS 112, 7713-7718. doi: 10.1073/pnas.1504615112

Brandman, O., and Hegde, R. S. (2016). Ribosome-associated protein quality control. Nat. Struct. Mol. Biol. 23, 7-15. doi: 10.1038/nsmb.3147

Brandman, O., Stewart-Ornstein, J., Wong, D., Larson, A., Williams, C. C., Li, G.-W., et al. (2012). A ribosome-bound quality control complex triggers degradation of nascent peptides and signals translation stress. Cell 151, 10421054. doi: 10.1016/j.cell.2012.10.044

Braun, R. J., and Westermann, B. (2017). With the help of MOM: mitochondrial contributions to cellular quality control. Trends Cell Biol. 27, 441-452. doi: 10.1016/j.tcb.2017.02.007

Chacinska, A., Koehler, C. M., Milenkovic, D., Lithgow, T., and Pfanner, N. (2009). Importing mitochondrial proteins: machineries and mechanisms. Cell 138, 628-644. doi: 10.1016/j.cell.2009.08.005

Chan, N. C., Salazar, A. M., Pham, A. H., Sweredoski, M. J., Kolawa, N. J., Graham, R. L. J., et al. (2011). Broad activation of the ubiquitin-proteasome system by Parkin is critical for mitophagy. Hum. Mol. Genet. 20, 1726-1737. doi: $10.1093 / \mathrm{hmg} / \mathrm{ddr} 048$

Chen, X., Yao, J.-M., Fang, X., Zhang, C., Yang, Y.-S., Hu, C.-P., et al. (2019). Hypoxia promotes pulmonary vascular remodeling via HIF-1 $\alpha$ to regulate mitochondrial dynamics. J. Geriatr. Cardiol. 16, 855-871.

Chen, Y.-C., Umanah, G. K. E., Dephoure, N., Andrabi, S. A., Gygi, S. P., Dawson, T. M., et al. (2014). Msp1/ATAD1 maintains mitochondrial function by facilitating the degradation of mislocalized tail-anchored proteins. EMBO J. 33, 1548-1564. doi: 10.15252/embj.201487943

Clague, M. J., Urbé, S., and Komander, D. (2019). Breaking the chains: deubiquitylating enzyme specificity begets function. Nat. Rev. Mol. Cell Biol. 20, 338-352. doi: 10.1038/s41580-019-0099-1

Cohen, M. M., Amiott, E. A., Day, A. R., Leboucher, G. P., Pryce, E. N., Glickman, M. H., et al. (2011). Sequential requirements for the GTPase domain of the mitofusin Fzo1 and the ubiquitin ligase SCFMdm30 in mitochondrial outer membrane fusion. J. Cell. Sci. 124, 1403-1410. doi: 10.1242/jcs.079293

Cohen, M. M. J., Leboucher, G. P., Livnat-Levanon, N., Glickman, M. H., and Weissman, A. M. (2008). Ubiquitin-proteasome-dependent degradation of 
a mitofusin, a critical regulator of mitochondrial fusion. Mol. Biol. Cell 19, 2457-2464. doi: 10.1091/mbc.E08-02-0227

Contreras, L., Drago, I., Zampese, E., and Pozzan, T. (2010). Mitochondria: the calcium connection. Biochim. Biophys. Acta 1797, 607-618. doi: 10.1016/j. bbabio.2010.05.005

Couvillion, M. T., Soto, I. C., Shipkovenska, G., and Churchman, L. S. (2016). Synchronized mitochondrial and cytosolic translation programs. Nature 533, 499-503. doi: 10.1038/nature18015

D'Amico, D., Sorrentino, V., and Auwerx, J. (2017). Cytosolic proteostasis networks of the mitochondrial stress response. Trends Biochem. Sci. 42, 712725. doi: 10.1016/j.tibs.2017.05.002

Dederer, V., Khmelinskii, A., Huhn, A. G., Okreglak, V., Knop, M., and Lemberg, M. K. (2019). Cooperation of mitochondrial and ER factors in quality control of tail-anchored proteins. eLife 8, 1-23. doi: 10.7554/eLife.45506

Defenouillère, Q., Yao, Y., Mouaikel, J., Namane, A., Galopier, A., Decourty, L., et al. (2013). Cdc48-associated complex bound to $60 \mathrm{~S}$ particles is required for the clearance of aberrant translation products. Proc. Natl. Acad. Sci. U.S.A 110, 5046-5051. doi: 10.1073/pnas. 1221724110

Denisenko, T. V., Gorbunova, A. S., and Zhivotovsky, B. (2019). Mitochondrial involvement in migration, invasion and metastasis. Front. Cell Dev. Biol. 7:355. doi: $10.3389 /$ fcell.2019.00355

Dikic, I. (2017). Proteasomal and autophagic degradation systems. Annu. Rev. Biochem. 86, 193-224. doi: 10.1146/annurev-biochem-061516-044908

Esaki, M., and Ogura, T. (2012). Cdc48p/p97-mediated regulation of mitochondrial morphology is Vms1p-independent. J. Struct. Biol. 179, 112-120. doi: 10.1016/j.jsb.2012.04.017

Escobar-Henriques, M., Altin, S., and Brave, F. D. (2020). Interplay between the ubiquitin proteasome system and mitochondria for protein homeostasis. Curr. Issues Mol. Biol. 35, 35-58. doi: 10.21775/cimb.035.035

Escobar-Henriques, M., and Anton, F. (2013). Mechanistic perspective of mitochondrial fusion: tubulation vs. fragmentation. Biochim. Biophys. Acta 1833, 162-175. doi: 10.1016/j.bbamcr.2012.07.016

Escobar-Henriques, M., Westermann, B., and Langer, T. (2006). Regulation of mitochondrial fusion by the F-box protein Mdm30 involves proteasomeindependent turnover of Fzo1. J. Cell Biol. 173, 645-650. doi: 10.1083/jcb. 200512079

Fiorese, C. J., Schulz, A. M., Lin, Y. F., Rosin, N., Pellegrino, M. W., and Haynes, C. M. (2016). The transcription factor ATF5 mediates a mammalian mitochondrial UPR. Curr. Biol. 26, 2037-2043. doi: 10.1016/j.cub.2016.06.002

Franz, A., Ackermann, L., and Hoppe, T. (2014). Create and preserve: proteostasis in development and aging is governed by Cdc48/p97/VCP. Biochim. Biophys. Acta 1843, 205-215. doi: 10.1016/j.bbamcr.2013.03.031

Franz, A., Kevei, E., and Hoppe, T. (2015). Double-edged alliance: mitochondrial surveillance by the UPS and autophagy. Curr. Opin. Cell Biol. 37, 18-27. doi: 10.1016/j.ceb.2015.08.004

Fritz, S., Weinbach, N., and Westermann, B. (2003). Mdm30 is an F-box protein required for maintenance of fusion-competent mitochondria in yeast. $M B o C$ 14, 2303-2313. doi: 10.1091/mbc.e02-12-0831

Glynn, S. E. (2017). Multifunctional mitochondrial AAA proteases. Front. Mol. Biosci. 4:34. doi: 10.3389/fmolb.2017.00034

Grune, T., Catalgol, B., Licht, A., Ermak, G., Pickering, A. M., Ngo, J. K., et al. (2011). HSP70 mediates dissociation and reassociation of the $26 \mathrm{~S}$ proteasome during adaptation to oxidative stress. Free Radic. Biol. Med. 51, 1355-1364. doi: 10.1016/j.freeradbiomed.2011.06.015

Guerriero, C. J., and Brodsky, J. L. (2012). The delicate balance between secreted protein folding and endoplasmic reticulum-associated degradation in human physiology. Physiol. Rev. 92, 537-576. doi: 10.1152/physrev.00027.2011

Haakonsen, D. L., and Rape, M. (2019). Branching out: improved signaling by heterotypic ubiquitin chains. Trends Cell Biol. 29, 704-716. doi: 10.1016/j.tcb. 2019.06.003

Hansen, K. G., Aviram, N., Laborenz, J., Bibi, C., Meyer, M., Spang, A., et al. (2018). An ER surface retrieval pathway safeguards the import of mitochondrial membrane proteins in yeast. Science 361, 1118-1122. doi: 10.1126/science. aar8174

Haynes, C. M., Yang, Y., Blais, S. P., Neubert, T. A., and Ron, D. (2010). The matrix peptide exporter HAF-1 signals a mitochondrial unfolded protein response by activating the transcription factor ZC376.7 in C. elegans. Mol. Cell 37, 529-540. doi: 10.1016/j.molcel.2010.01.015
Helle, S. C. J., Kanfer, G., Kolar, K., Lang, A., Michel, A. H., and Kornmann, B. (2013). Organization and function of membrane contact sites. Biochim. Biophys. Acta 1833, 2526-2541. doi: 10.1016/j.bbamcr.2013.01.028

Heo, J. M., Livnat-Levanon, N., Taylor, E. B., Jones, K. T., Dephoure, N., Ring, J., et al. (2010). A stress-responsive system for mitochondrial protein degradation. Mol. Cell 40, 465-480. doi: 10.1016/j.molcel.2010.10.021

Heo, J.-M., and Rutter, J. (2011). Ubiquitin-dependent mitochondrial protein degradation. Int. J. Biochem. Cell Biol. 43, 1422-1426.

Inuzuka, H., Shaik, S., Onoyama, I., Gao, D., Tseng, A., Maser, R. S., et al. (2011). SCF FBW7 regulates cellular apoptosis by targeting MCL1 for ubiquitylation and destruction. Nature 471, 104-109. doi: 10.1038/nature 09732

Itakura, E., Zavodszky, E., Shao, S., Wohlever, M. L., Keenan, R. J., and Hegde, R. S. (2016). Ubiquilins chaperone and triage mitochondrial membrane proteins for degradation. Mol. Cell 63, 21-33. doi: 10.1016/j.molcel.2016.05.020

Izawa, T., Park, S.-H., Zhao, L., Hartl, F. U., and Neupert, W. (2017). Cytosolic protein vms1 links ribosome quality control to mitochondrial and cellular homeostasis. Cell 171, 890-903.e18. doi: 10.1016/j.cell.2017.10.002

Joazeiro, C. A. P. (2019). Mechanisms and functions of ribosome-associated protein quality control. Nat. Rev. Mol. Cell Biol. 20, 368-383. doi: 10.1038/ s41580-019-0118-2

Karbowski, M., Neutzner, A., and Youle, R. J. (2007). The mitochondrial E3 ubiquitin ligase MARCH5 is required for Drp1 dependent mitochondrial division. J. Cell Biol. 178, 71-84. doi: 10.1083/jcb.200611064

Kaushik, S., and Cuervo, A. M. (2015). Proteostasis and aging. Nat. Med. 21, 1406-1415.

Kerscher, O., Felberbaum, R., and Hochstrasser, M. (2006). Modification of proteins by ubiquitin and ubiquitin-like proteins. Annu. Rev. Cell Dev. Biol. 22, 159-180. doi: 10.1146/annurev.cellbio.22.010605.093503

Khaminets, A., Behl, C., and Dikic, I. (2016). Ubiquitin-dependent and independent signals in selective autophagy. Trends Cell Biol. 26, 6-16. doi: 10.1016/j.tcb.2015.08.010

Kim, D. K., and Mook-Jung, I. (2019). The role of cell type-specific mitochondrial dysfunction in the pathogenesis of Alzheimer's disease. BMB Rep. 52, 679-688. doi: 10.5483/bmbrep.2019.52.12.282

Kim, N. C., Tresse, E., Kolaitis, R.-M., Molliex, A., Thomas, R. E., Alami, N. H., et al. (2013). VCP is essential for mitochondrial quality control by PINK1/parkin and this function is impaired by VCP mutations. Neuron 78, 65-80. doi: 10.1016/j. neuron.2013.02.029

Klaips, C. L., Jayaraj, G. G., and Hartl, F. U. (2018). Pathways of cellular proteostasis in aging and disease. J. Cell Biol. 217, 51-63. doi: 10.1083/jcb.201709072

Koppen, M., and Langer, T. (2007). Protein degradation within mitochondria: versatile activities of AAA proteases and other peptidases. Crit. Rev. Biochem. Mol. Biol. 42, 221-242. doi: 10.1080/10409230701380452

Kowalski, L., Bragoszewski, P., Khmelinskii, A., Glow, E., Knop, M., and Chacinska, A. (2018). Determinants of the cytosolic turnover of mitochondrial intermembrane space proteins. BMC Biol. 16:66. doi: 10.1186/s12915-0180536- 1

Lackner, L. L. (2014). Shaping the dynamic mitochondrial network. BMC Biol. 12:35. doi: 10.1186/1741-7007-12-35

Lefaki, M., Papaevgeniou, N., and Chondrogianni, N. (2017). Redox regulation of proteasome function. Redox. Biol. 13, 452-458. doi: 10.1016/j.redox.2017. 07.005

Lill, R., and Mühlenhoff, U. (2008). Maturation of iron-sulfur proteins in eukaryotes: mechanisms, connected processes, and diseases. Annu. Rev. Biochem. 77, 669-700. doi: 10.1146/annurev.biochem.76.052705.162653

Livnat-Levanon, N., Kevei, E., Kleifeld, O., Krutauz, D., Segref, A., Rinaldi, T., et al. (2014). Reversible 26S proteasome disassembly upon mitochondrial stress. Cell Rep. 7, 1371-1380. doi: 10.1016/j.celrep.2014.04.030

Lu, K., den Brave, F., and Jentsch, S. (2017). Receptor oligomerization guides pathway choice between proteasomal and autophagic degradation. Nat. Cell Biol. 19, 732-739. doi: 10.1038/ncb3531

Marshall, R. S., and Vierstra, R. D. (2019). Dynamic regulation of the 26S proteasome: from synthesis to degradation. Front. Mol. Biosci. 6:40. doi: 10. 3389/fmolb.2019.00040

Mårtensson, C. U., Priesnitz, C., Song, J., Ellenrieder, L., Doan, K. N., Boos, F., et al. (2019). Mitochondrial protein translocation-associated degradation. Nature 569, 679-683. doi: 10.1038/s41586-019-1227-y 
Martinus, R. D., Garth, G. P., Webster, T. L., Cartwright, P., Naylor, D. J., Høj, P. B., et al. (1996). Selective induction of mitochondrial chaperones in response to loss of the mitochondrial genome. Eur. J. Biochem. 240, 98-103. doi: 10.1111/ j.1432-1033.1996.0098h.x

Matsumoto, S., Nakatsukasa, K., Kakuta, C., Tamura, Y., Esaki, M., and Endo, T. (2019). Mspl clears mistargeted proteins by facilitating their transfer from mitochondria to the ER. Mol. Cell 76, 191.e-205.e. doi: 10.1016/j.molcel.2019. 07.006

Mehrtash, A. B., and Hochstrasser, M. (2018). Ubiquitin-dependent protein degradation at the endoplasmic reticulum and nuclear envelope. Semin. Cell Dev. Biol. 93, 111-124. doi: 10.1016/j.semcdb.2018.09.013

Moltedo, O., Remondelli, P., and Amodio, G. (2019). The mitochondriaendoplasmic reticulum contacts and their critical role in aging and ageassociated diseases. Front. Cell Dev. Biol. 7:172. doi: 10.3389/fcell.2019.00172

Mouysset, J., Kähler, C., and Hoppe, T. (2006). A conserved role of caenorhabditis elegans CDC-48 in ER-associated protein degradation. J. Struct. Biol. 156, 41-49. doi: 10.1016/j.jsb.2006.02.015

Münch, C. (2018). The different axes of the mammalian mitochondrial unfolded protein response. BMC Biol. 16:81. doi: 10.1186/s12915-018-0548-x

Nakai, M., Endo, T., Hase, T., and Matsubara, H. (1993). Intramitochondrial protein sorting. Isolation and characterization of the yeast MSP1 gene which belongs to a novel family of putative ATPases. J. Biol. Chem. 268, 24262-24269.

Nargund, A. M., Pellegrino, M. W., Fiorese, C. J., Baker, B. M., and Haynes, C. M. (2012). Mitochondrial import efficiency of ATFS-1 regulates mitochondrial UPR activation. Science 337, 587-590. doi: 10.1126/science.12 23560

Neuber, O., Jarosch, E., Volkwein, C., Walter, J., and Sommer, T. (2005). Ubx2 links the Cdc48 complex to ER-associated protein degradation. Nat. Cell Biol. 7, 993-998. doi: 10.1038/ncb1298

Neupert, W., and Herrmann, J. M. (2007). Translocation of proteins into mitochondria. Annu. Rev. Biochem. 76, 723-749. doi: 10.1146/annurev. biochem.76.052705.163409

Neutzner, A., and Youle, R. J. (2005). Instability of the mitofusin Fzo1 regulates mitochondrial morphology during the mating response of the yeast Saccharomyces cerevisiae. J. Biol. Chem. 280, 18598-18603. doi: 10.1074/jbc. m500807200

Okreglak, V., and Walter, P. (2014). The conserved AAA-ATPase Msp1 confers organelle specificity to tail-anchored proteins. Proc. Natl. Acad. Sci. U.S.A. 111, 8019-8024. doi: 10.1073/pnas.1405755111

Pakos-Zebrucka, K., Koryga, I., Mnich, K., Ljujic, M., Samali, A., and Gorman, A. M. (2016). The integrated stress response. EMBO Rep. 17, 1374-1395.

Palikaras, K., Lionaki, E., and Tavernarakis, N. (2018). Mechanisms of mitophagy in cellular homeostasis, physiology and pathology. Nat. Cell Biol. 20, 1013-1022. doi: 10.1038/s41556-018-0176-2

Papa, L., and Germain, D. (2011). Estrogen receptor mediates a distinct mitochondrial unfolded protein response. J. Cell Sci. 124, 1396-1402. doi: 10. 1242/jcs.078220

Park, Y.-Y., Lee, S., Karbowski, M., Neutzner, A., Youle, R. J., and Cho, H. (2010). Loss of MARCH5 mitochondrial E3 ubiquitin ligase induces cellular senescence through dynamin-related protein 1 and mitofusin 1. J. Cell Sci. 123, 619-626. doi: $10.1242 /$ jcs.061481

Pfanner, N., Warscheid, B., and Wiedemann, N. (2019). Mitochondrial proteins: from biogenesis to functional networks. Nat. Rev. Mol. Cell Biol. 20, 267-284. doi: 10.1038/s41580-018-0092-0

Phillips, M. J., and Voeltz, G. K. (2016). Structure and function of ER membrane contact sites with other organelles. Nat. Rev. Mol. Cell Biol. 17, 69-82. doi: 10.1038/nrm.2015.8

Pickles, S., Vigié, P., and Youle, R. J. (2018). Mitophagy and quality control mechanisms in mitochondrial maintenance. Curr. Biol. 28, R170-R185.

Pohl, C., and Dikic, I. (2019). Cellular quality control by the ubiquitin-proteasome system and autophagy. Science 366, 818-822. doi: 10.1126/science.aax3769

Quirós, P. M., Langer, T., and López-Otín, C. (2015). New roles for mitochondrial proteases in health, ageing and disease. Nat. Rev. Mol. Cell Biol. 16, 345-359. doi: $10.1038 / \mathrm{nrm} 3984$

Quirós, P. M., Prado, M. A., Zamboni, N., D’Amico, D., Williams, R. W., Finley, D., et al. (2017). Multi-omics analysis identifies ATF4 as a key regulator of the mitochondrial stress response in mammals. J. Cell Biol. 216, 2027-2045. doi: $10.1083 /$ jcb. 201702058
Radke, S., Chander, H., Schäfer, P., Meiss, G., Krüger, R., Schulz, J. B., et al. (2008). Mitochondrial protein quality control by the proteasome involves ubiquitination and the protease omi. J. Biol. Chem. 283, 12681-12685. doi: 10.1074/jbc.C800036200

Raturi, A., and Simmen, T. (2013). Where the endoplasmic reticulum and the mitochondrion tie the knot: the mitochondria-associated membrane (MAM). Biochim. Biophys. Acta 1833, 213-224. doi: 10.1016/j.bbamcr.2012.04.013

Rendón, O. Z., Fredrickson, E. K., Howard, C. J., Vranken, J. V., Fogarty, S., Tolley, N. D., et al. (2018). Vmslp is a release factor for the ribosome-associated quality control complex. Nat. Commun. 9, 1-9. doi: 10.1038/s41467-018-04564-3

Richter, K., Haslbeck, M., and Buchner, J. (2010). The heat shock response: life on the verge of death. Mol. Cell 40, 253-266. doi: 10.1016/j.molcel.2010.10.006

Roy, M., Reddy, P. H., Iijima, M., and Sesaki, H. (2015). Mitochondrial division and fusion in metabolism. Curr. Opin. Cell Biol. 33, 111-118. doi: 10.1016/j.ceb. 2015.02.001

Ruan, L., Zhou, C., Jin, E., Kucharavy, A., Zhang, Y., Wen, Z., et al. (2017). Cytosolic proteostasis through importing of misfolded proteins into mitochondria. Nature 543, 443-446. doi: 10.1038/nature21695

Ruggiano, A., Foresti, O., and Carvalho, P. (2014). Quality control: ER-associated degradation: protein quality control and beyond. J. Cell Biol. 204, 869-879. doi: $10.1083 /$ jcb. 201312042

Samluk, L., Chroscicki, P., and Chacinska, A. (2018). Mitochondrial protein import stress and signaling. Curr. Opin. Physiol. 3, 41-48. doi: 10.1016/j.cophys.2018. 02.010

Sauer, R. T., and Baker, T. A. (2011). AAA+ proteases: ATP-fueled machines of protein destruction. Annu. Rev. Biochem. 80, 587-612. doi: 10.1146/annurevbiochem-060408-172623

Schuberth, C., and Buchberger, A. (2005). Membrane-bound Ubx2 recruits Cdc48 to ubiquitin ligases and their substrates to ensure efficient ER-associated protein degradation. Nat. Cell Biol. 7, 999-1006. doi: 10.1038/ncb1299

Segref, A., Kevei, E., Pokrzywa, W., Schmeisser, K., Mansfeld, J., Livnat-Levanon, N., et al. (2014). Pathogenesis of human mitochondrial diseases is modulated by reduced activity of the ubiquitin/proteasome system. Cell Metab. 19, 642-652. doi: 10.1016/j.cmet.2014.01.016

Shao, S., von der Malsburg, K., and Hegde, R. S. (2013). Listerin-dependent nascent protein ubiquitination relies on ribosome subunit dissociation. Mol. Cell 50, 637-648. doi: 10.1016/j.molcel.2013.04.015

Shpilka, T., and Haynes, C. M. (2018). The mitochondrial UPR: mechanisms, physiological functions and implications in ageing. Nat. Rev. Mol. Cell Biol. 19, 109-120. doi: 10.1038/nrm.2017.110

Simões, T., Schuster, R., den Brave, F., and Escobar-Henriques, M. (2018). Cdc48 regulates a deubiquitylase cascade critical for mitochondrial fusion. eLife 7:e30015. doi: 10.7554/eLife.30015

Sonenberg, N., and Hinnebusch, A. G. (2009). Regulation of translation initiation in eukaryotes: mechanisms and biological targets. Cell 136, 731-745. doi: 10. 1016/j.cell.2009.01.042

Suhm, T., Kaimal, J. M., Dawitz, H., Peselj, C., Masser, A. E., Hanzén, S., et al. (2018). Mitochondrial translation efficiency controls cytoplasmic protein homeostasis. Cell Metab. 27, 1309-1322.e6. doi: 10.1016/j.cmet.2018.04.011

Tanaka, A., Cleland, M. M., Xu, S., Narendra, D. P., Suen, D.-F., Karbowski, M., et al. (2010). Proteasome and p97 mediate mitophagy and degradation of mitofusins induced by ParkinPINK1- and Parkin-mediated proteasomal degradation of Mfn1 and Mfn2. J. Cell Biol. 191, 1367-1380. doi: 10.1083/jcb. 201007013

Tapias, V. (2019). Editorial: mitochondrial dysfunction and neurodegeneration. Front. Neurosci. 13:1372. doi: 10.3389/fnins.2019.01372

Topf, U., Suppanz, I., Samluk, L., Wrobel, L., Böser, A., Sakowska, P., et al. (2018). Quantitative proteomics identifies redox switches for global translation modulation by mitochondrially produced reactive oxygen species. Nat. Commun. 9, 1-17.

Topf, U., Wrobel, L., and Chacinska, A. (2016). Chatty mitochondria: keeping balance in cellular protein homeostasis. Trends Cell Biol. 26, 577-586. doi: 10.1016/j.tcb.2016.03.002

van Vliet, A. R., Verfaillie, T., and Agostinis, P. (2014). New functions of mitochondria associated membranes in cellular signaling. Biochim Biophys. Acta 1843, 2253-2262. doi: 10.1016/j.bbamcr.2014.03.009

Verma, R., Reichermeier, K. M., Burroughs, A. M., Oania, R. S., Reitsma, J. M., Aravind, L., et al. (2018). Vms1 and ANKZF1 peptidyl-tRNA hydrolases release 
nascent chains from stalled ribosomes. Nature 557, 446-451. doi: 10.1038/ s41586-018-0022-5

Vyas, S., Zaganjor, E., and Haigis, M. C. (2016). Mitochondria and cancer. Cell 166, 555-566.

Wai, T., and Langer, T. (2016). Mitochondrial dynamics and metabolic regulation. Trends Endocrinol. Metab. 27, 105-117. doi: 10.1016/j.tem.2015. 12.001

Walter, P., and Ron, D. (2011). The unfolded protein response: from stress pathway to homeostatic regulation. Science 334, 1081-1086. doi: 10.1126/ science. 1209038

Wang, C., and Youle, R. J. (2009). The role of mitochondria in apoptosis. Annu. Rev. Genet. 43, 95-118. doi: 10.1146/annurev-genet-102108-134850

Wang, X., and Chen, X. J. (2015). A cytosolic network suppressing mitochondriamediated proteostatic stress and cell death. Nature 524, 481-484. doi: 10.1038/ nature 14859

Wang, X., Yen, J., Kaiser, P., and Huang, L. (2010). Regulation of the $26 \mathrm{~S}$ proteasome complex during oxidative stress. Sci. Signal. 3:ra88. doi: 10.1126/ scisignal.2001232

Wang, Z.-H., Clark, C., and Geisbrecht, E. R. (2016). Drosophila clueless is involved in Parkin-dependent mitophagy by promoting VCP-mediated marf degradation. Hum. Mol. Genet. 25, 1946-1964. doi: 10.1093/hmg/ddw067

Weidberg, H., and Amon, A. (2018). MitoCPR-a surveillance pathway that protects mitochondria in response to protein import stress. Science 360:eaan4146. doi: 10.1126/science.aan4146

Weir, N. R., Kamber, R. A., Martenson, J. S., and Denic, V. (2017). The AAA protein Mspl mediates clearance of excess tail-anchored proteins from the peroxisomal membrane. eLife 6:e28507.

Whiteley, A. M., Prado, M. A., Peng, I., Abbas, A. R., Haley, B., Paulo, J. A., et al. (2017). Ubiquilin1 promotes antigen-receptor mediated proliferation by eliminating mislocalized mitochondrial proteins. eLife 6:e26435. doi: 10.7554/ eLife. 26435

Wiedemann, N., and Pfanner, N. (2017). Mitochondrial machineries for protein import and assembly. Annu. Rev. Biochem. 86, 685-714. doi: 10.1146/annurevbiochem-060815-014352

Wohlever, M. L., Mateja, A., McGilvray, P. T., Day, K. J., and Keenan, R. J. (2017). Mspl is a membrane protein dislocase for tail-anchored proteins. Mol. Cell 67, 194-202.e6. doi: 10.1016/j.molcel.2017.06.019
Wrobel, L., Topf, U., Bragoszewski, P., Wiese, S., Sztolsztener, M. E., Oeljeklaus, S., et al. (2015). Mistargeted mitochondrial proteins activate a proteostatic response in the cytosol. Nature 524, 485-488. doi: 10.1038/nature14951

Wu, X., Li, L., and Jiang, H. (2016). Doal targets ubiquitinated substrates for mitochondria-associated degradation. J. Cell Biol. 213, 49-63. doi: 10.1083/jcb. 201510098

Xu, S., Peng, G., Wang, Y., Fang, S., and Karbowsk, M. (2011). The AAA-ATPase p97 is essential for outer mitochondrial membrane protein turnover. Mol. Biol. Cell 22, 291-300. doi: 10.1091/mbc.e10-09-0748

Yonashiro, R., Ishido, S., Kyo, S., Fukuda, T., Goto, E., Matsuki, Y., et al. (2006). A novel mitochondrial ubiquitin ligase plays a critical role in mitochondrial dynamics. EMBO J. 25, 3618-3626. doi: 10.1038/sj.emboj.7601249

Yoneda, T. (2004). Compartment-specific perturbation of protein handling activates genes encoding mitochondrial chaperones. J. Cell Sci. 117, 4055-4066. doi: $10.1242 /$ jcs. 01275

Youle, R. J. (2019). Mitochondria-striking a balance between host and endosymbiont. Science 365:eaaw9855. doi: 10.1126/science.aaw9855

Zhao, Q., Wang, J., Levichkin, I. V., Stasinopoulos, S., Ryan, M. T., and Hoogenraad, N. J. (2002). A mitochondrial specific stress response in mammalian cells. EMBO J. 21, 4411-4419. doi: 10.1093/emboj/cdf445

Zheng, J., Li, L., and Jiang, H. (2019). Molecular pathways of mitochondrial outer membrane protein degradation. Biochem. Soc. Trans. 47, 1437-1447. doi: 10. 1042/bst20190275

Ziviani, E., Tao, R. N., and Whitworth, A. J. (2010). Drosophila Parkin requires PINK1 for mitochondrial translocation and ubiquitinates Mitofusin. PNAS 107, 5018-5023. doi: 10.1073/pnas.0913485107

Conflict of Interest: The authors declare that the research was conducted in the absence of any commercial or financial relationships that could be construed as a potential conflict of interest.

Copyright (c) 2020 Ravanelli, den Brave and Hoppe. This is an open-access article distributed under the terms of the Creative Commons Attribution License (CC BY). The use, distribution or reproduction in other forums is permitted, provided the original author(s) and the copyright owner(s) are credited and that the original publication in this journal is cited, in accordance with accepted academic practice. No use, distribution or reproduction is permitted which does not comply with these terms. 